EDITORIAL

\title{
Judicious use of transthoracic echocardiography in the diagnosis of infective endocarditis
}

\section{P Robles}

Heart 2003;89:1283-1284

\begin{abstract}
Patients with a very low clinical probability of infective endocarditis do not benefit from echocardiography-but what is the definition of "very low probability"?
\end{abstract}

$\mathrm{T}$ he diagnosis of infective endocarditis (IE) requires the integration of clinical, laboratory, and echocardiographic data. During the past two decades, various clinical criteria have been proposed and subsequently modified for the diagnosis of IE. In 1994 a group at Duke University proposed standardised criteria for assessing patients with suspected IE. ${ }^{1}$ These criteria integrated factors predisposing patients to the development of IE, the blood culture isolate and persistence of bacteraemia, and echocardiographic findings with other clinical and laboratory information. The usefulness of these Duke criteria in assessing patients with potential IE has been validated in several subsequent studies. ${ }^{2}$

Transthoracic echocardiography (TTE) is rapid and non-invasive and has excellent specificity for vegetations. According to the population studied, TTE has been reported to have a sensitivity of $40-80 \%$ for the detection of vegetations. ${ }^{3}$ Because of the possibility of a false negative examination (or the absence of a vegetation) or a false positive study (Lambl's excrescenses, non-infective vegetations, thrombi), echocardiography should not supplant clinical and microbiological diagnosis. TTE views may be inadequate in up to $20 \%$ of adult patients because of obesity, chronic obstructive pulmonary disease, or chest wall deformities. In patients suspected of having IE, TTE alone cannot exclude several important aspects of IE, vegetation, or inadequate views to detect small abscesses. ${ }^{4}$

Echocardiography is often requested in patients with fever who have a low probability of endocarditis, and is also requested in patients in whom the diagnosis is virtually certain. Echocardiography has some limitations. It is not cost effective as a means of excluding IE in patients with a low pretest probability of having the disease. With higher prior probability, a negative study result has a useful negative predictive value, but it cannot totally exclude the diagnosis of endocarditis. While most studies involving IE have focused on confirming the presence of the disease, there are few reports to date that have attempted to identify criteria that would predict the absence of vegetations on transthoracic echocardiography. ${ }^{5}$ Lindner and colleagues have evaluated the diagnostic value of TTE in suspected IE on the basis of the pretest probability of disease by using clinical criteria that differed from the Duke criteria. In this report, if this probability is less than $4 \%$, a negative transthoracic echocardiogram is cost effective and clinically satisfactory in ruling out IE. ${ }^{6}$ As a general rule, TTE with Doppler flow studies should be performed in anyone suspected of having endocarditis. If the clinical suspicion of endocarditis is high and the TTE study is negative or inconclusive, a transoesophageal echocardiogram should be obtained.

ENDOCARDITIS: A CLINICAL DIAGNOSIS Despite these advances in technology, endocarditis remains a clinical diagnosis. The diagnosis of IE is straightforward in those patients with classic oslerian manifestations: bacteraemia or fungaemia, evidence of active valvulitis, peripheral emboli, and immunologic vascular phenomena. In other patients, however, classic peripheral stigmata may be few or absent. The variability in the clinical presentation of IE requires a diagnostic strategy that will be both sensitive for disease detection and specific for its exclusion across all the forms of the disease.

In this cost conscious era the screening approach for patients with suspected endocarditis, described in Heart recently by Greaves and colleagues, is essential. ${ }^{7}$ According to the probability theory, a test should not offer additional information when the pretest likelihood of disease is low. Patients with a low clinical probability of IE do not benefit from echocardiography. Despite recent American Heart Association guidelines and other authors suggesting the use of transthoracic echocardiography is not indicated in patients with a very low probability of IE, nobody has defined the "very low probability" group.

However, Greaves and colleagues ${ }^{7}$ propose criteria which define this very low probability group successfully. This study set out to identify clinical criteria that might permit more judicious use of TTE in patients referred for echocardiography for suspicion of IE. These criteria were: (1) vasculitic/embolic phenomena; (2) central venous access; (3) a recent history of intravenous drug use; (4) presence of a prosthetic valve; and (5) positive blood cultures. The collective absence of these five criteria indicated a zero probability of IE being demonstrated on echocardiography.

Greaves and colleagues ${ }^{7}$ also provide information about the predictive value of TTE in suspected IE. The extent to which these data are applicable to the general population remains to be determined. Further prospective studies 
involving large numbers of patients will be required to resolve these issues and determine the most clinically useful variable for detecting the "very low probability" group.

\section{RISK OF DEVELOPING IE}

The risk of developing an episode of IE depends on factors related to the affected patient and to specific dental, surgical, and therapeutic procedures that cause transient bacteraemias from microorganisms commonly associated with IE. The predisposing factors (considered minor clinical criteria in the Duke model) include, in addition to intravenous drug addiction, valvar lesions of both the native valve (of congenital, rheumatic, or degenerative origin) and the prosthetic valve (biological or mechanical). The literature, on the other hand, describes numerous risk factors, such as poor dental hygiene, chronic alcoholism, and diseases which cause immunological changes such as systemic lupus erythematous, diabetes mellitus, renal insufficiency, cancer, or chronic inflammatory intestinal disease. Greaves and colleagues $^{7}$ highlight some important points about one additional predisposing factor-central venous access. These findings are in line with recent data from population based epidemiologic studies where nosocomial endocarditis is now estimated to account for around 10-15\% of all episodes of endocarditis. ${ }^{9}$ The localisation of infection to the right side of the heart may be related to the above possible risk factors, essentially pacemakers and central venous access. Although the addition of new criteria needs rigorous prospective evaluation, probably the existence of a central venous access should be considered as a new minor criterion in future modifications of Duke's scheme.

\section{REFERENCES}

1 Durack DT, Lukes AS, Bright DK. New criteria for diagnosis of infective endocarditis: utilisation of specific echocardiographic findings. Duke endocarditis service. Am J Med 1994;96:200-9.

2 Li JS, Sexton DJ, Mick N, et al. Proposed modifications to the Duke criteria for the diagnosis of infective endocarditis. Clin Infect Dis 2000;30:633-8.

3 Mylonakis E, Calderwood SB. Infective endocarditis in adults. N Engl J Med 2001;345:1318-30.

4 Bayer AS, Bolger AF, Taubert KA, et al. Diagnosis and management of infective endocarditis and its complications. Circulation 1998;98:2936-48.

5 Heidenreich PA, Masoudi FA, Maini B, et al. Echocardiography in patients with suspected endocarditis: a cost effective analysis. Am J Med 1999; 107:198-208.

6 Lindner JR, Case RA, Dent JM, et al. Diagnostic value of echocardiography in suspected endocarditis. An evaluation based on the pre-test probability of disease. Circulation 1996:93:730-6.

7 Greaves K, Mou D, Patel A, et al. Clinical criteria and the appropriate use of transthoracic echocardiography for the exclusion of infective endocarditis. Heart 2003:89:273-5.

8 Cheitlin MD, Alpert JS, Armstrong WF, et al. ACC/AHA guidelines for the clinical application of echocardiography: Executive summary: a report of the American College of Cardiology/American Heart Association task force on practice guidelines (committee on clinical application of echocardiography). Developed in collaboration with the American Society of Echocardiography. J Am Coll Cardiol 1997;29:862-79.

9 Fernandez Guerrero ML, Verdejo C, Azofra J, et al. Hospital adquired infectious endocarditis not associated with cardiac surgery: an emerging problem. Clin Infect Dis 1995;20:16-23.

\section{FROM BMJ JOURNALS}

\section{Factors associated with coronary artery calcification in young female patients with SLE}

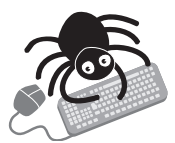

Please visit the Heart website [www.heartinl. com] for a link to the full text of this article.
K Manger, M Kusus, C Forster, D Ropers, W G Daniel, J R Kalden, S Achenbach, B Manger

Background: With improved survival rates of patients with systemic lupus erythematosus (SLE), damage such as accelerated atherosclerosis gains increasing importance. Objective: To identify the prevalence of coronary artery calcifications (CAC) in asymptomatic patients.

Methods: Electron beam tomography (EBT) was performed in 75 female patients with SLE aged $<50$. The results were correlated with traditional and SLE related factors associated with CAC. 49 women with symptomatic coronary heart disease (CHD) and 279 women without CHD were also analysed.

Results: Overall, 21/75 (28\%) patients had CAC. Low HDL cholesterol levels < $1.40 \mathrm{mmol} / \mathrm{l}$ $(\mathrm{p}=0.03, \mathrm{OR}=1.8,67 \% \mathrm{v} 39 \%)$ and cigarette smoking $(\mathrm{p}=0.01, \mathrm{OR}=5.7,76 \% \mathrm{v} 44 \%)$ were identified as factors associated with CAC. Hypertension and high cholesterol were more common in women with CHD $(\mathrm{p}<0.01)$ than in those without CHD. SLE related factors were proteinuria ( $1331 \mathrm{v} 465 \mathrm{mg} /$ day, $\mathrm{p}=0.02)$, impaired renal function $(\mathrm{p}=0.02, \mathrm{OR}=2.6$, $26 \%$ v $6 \%)$, and high $\mathrm{C} 3$ levels $(\mathrm{p}=0.04, \mathrm{OR}=1.8,65 \%$ v $38 \%)$. High $\mathrm{C} 3$ levels were also more common in symptomatic CHD $(\mathrm{p}=0.02)$. The prevalence of Sm antibodies was lower in patients with CAC $(15 \% \mathrm{v} 42 \%, \mathrm{p}=0.03)$. In a multivariate analysis, cigarette smoking, reduced renal function, high $\mathrm{C} 3$, and a cumulative steroid dose above $30 \mathrm{~g}$ were the most important CAC associated factors in the lupus cohort.

Conclusion: A subgroup of patients with SLE with CAC without any clinical symptoms of CHD was identified by EBT. Therefore, EBT is useful for assessing asymptomatic atherosclerosis in this group.

A Annals of the Rheumatic Diseases 2003;62:846-850. 